\title{
Evolutionary models of binaries
}

\section{Walter van Rensbergen, Nicki Mennekens, Jean-Pierre de Greve, Kim Jansen and Bert de Loore}

\author{
Astrophysical Institute, Vrije Universiteit Brussel \\ Pleinlaan 2, B-1050 Brussels, Belgium \\ email: wvanrens@vub.ac.be, nmenneke@vub.ac.be, jpdgreve@vub.ac.be, \\ kim.jansen@telenet.be, cdeloore@pandora.be
}

\begin{abstract}
We have put on CDS a catalog containing 561 evolutionary models of binaries: $\mathrm{J} / \mathrm{A}+\mathrm{A} / 487 / 1129$ (Van Rensbergen+, 2008). The catalog covers a grid of binaries with a Btype primary at birth, different values for the initial mass ratio and a wide range of initial orbital periods. The evolution was calculated with the Brussels code in which we introduced the spinning up and the creation of a hot spot on the gainer or its accretion disk, caused by impacting mass coming from the donor. When the kinetic energy of fast rotation added to the radiative energy of the hot spot exceeds the binding energy, a fraction of the transferred matter leaves the system: the evolution is liberal during a short lasting era of rapid mass transfer. The spin-up of the gainer was modulated using both strong and weak tides. The catalog shows the results for both types. For comparison, we included the evolutionary tracks calculated with the conservative assumption. Binaries with an initial primary below $6 M_{\odot}$ show hardly any mass loss from the system and thus evolve conservatively. Above this limit differences between liberal and conservative evolution grow with increasing initial mass of the primary star.
\end{abstract}

Keywords. binaries: general, stars: evolution, stars: mass loss, stars: variables: other, stars: statistics, stars: fundamental parameters, catalogs

\section{Introduction}

Codes calculating conservative evolution of binaries have been developed by e.g. Paczyński (1967a,b), Kippenhahn, \& Weigert (1967), Kippenhahn et al. (1967) and Nelson \& Eggleton (2001). Mass loss during liberal evolution is defined by a quantity $\beta$ giving the fraction of mass lost by the donor (subscript $d$ ) that is accreted by the gainer (subscript $g$ ):

$$
\dot{M}_{g}=-\beta \dot{M}_{d} \quad \text { with } 0 \leqslant \beta \leqslant 1
$$

Conservative evolution is thus characterized by $\beta=1$. Liberal evolution further depends on the amount of angular momentum which is taken away by the matter that leaves the system. This is characterized by Podsiadlowski et al. (1992) as a quantity $\alpha$, determined by the location where the mass leaves the system. Our liberal code assumes that matter is lost from the hot spot on the gainer (or accretion disk around it) so that the escaping matter removes only the angular momentum of the gainer's orbit. In that case one obtains a time dependent value of $\alpha$ :

$$
\alpha=\left(\frac{M_{d}}{M_{g}+M_{d}}\right)^{2}
$$

A typical value of $\alpha$ during the fast and liberal era of Roche lobe overflow during $\mathrm{H}$ core burning of the donor (RLOF $\mathrm{A})$ is then $\alpha \approx 0.25(\mathrm{q} \approx 1)$. When this liberal era is succeeded by a fast and liberal era of RLOF $\mathrm{B}$ during $\mathrm{H}$ shell burning, the value of $\alpha$ turns out to be much smaller and can easily be calculated from relation (1.2). 


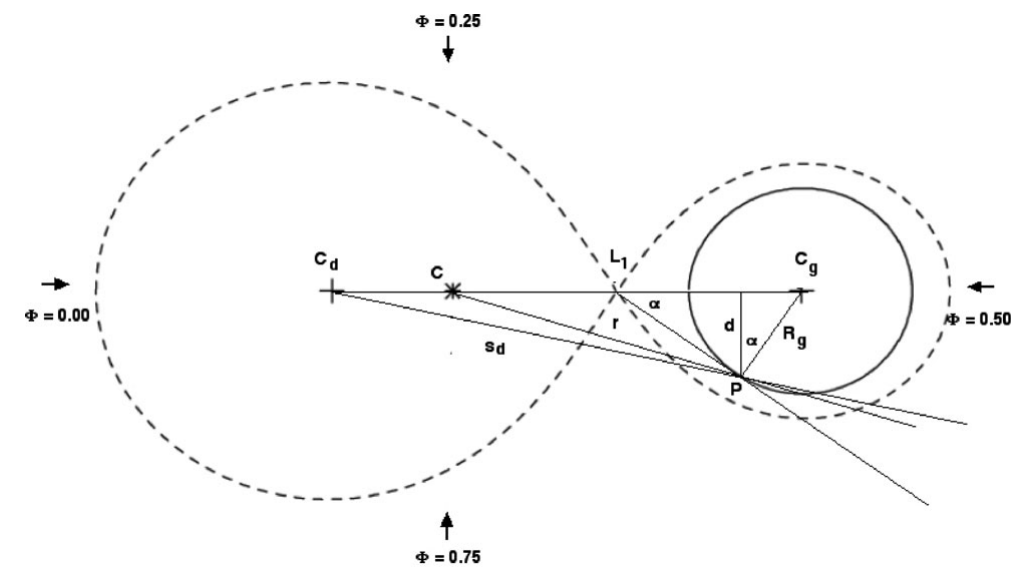

Figure 1. Geometry of a semi-detached binary, showing the impact parameter $d$ and the impact point $P$ at the equator of the gainer or at the edge of its accretion disk. The lines of sight at different orbital phases are indicated by arrows.

Values of $\beta<1$ were proposed by Meurs \& Van den Heuvel (1989) in order to theoretically reproduce the observed numbers of persistent strong massive binary X-ray sources and the number of observed Wolf-Rayet binaries. Binary evolutionary calculations with constant values of $\beta$ (e.g. $\beta=0.5$ ) were then reproduced by various authors, e.g. De Loore \& De Greve (1992).

Although mass loss from binaries is needed in evolutionary theory, it would be astonishing that $\beta$ does not depend on the mass transfer rate. It is more plausible that evolution of semi-detached binaries remains conservative $(\beta=1)$ during the long lasting quiet eras of slow RLOF, and that mass can only be lost from the system $(\beta<1)$ during short lasting violent eras of rapid mass transfer.

Calculations yielding time dependent behavior of $\beta$ for massive binaries have been published by Wellstein et al. (2001), showing binary evolution which is conservative most of the time but severely liberal during epochs of fast mass transfer. A scenario for liberal evolution of binaries with an intermediate mass primary at birth was discussed by Van Rensbergen et al. (2008, 2010a,b). Spin-up and hot spots created on the gainer by mass transferred from the donor can drive mass out of a binary. The scenario is discussed in detail in this contribution.

\section{Geometry of the system}

Spin-up of the gainer and the creation of a hot spot on the gainer's equator are caused by the impact of RLOF-material starting from the first Lagrangian point $L_{1}$ and impacting at $P$ as is shown in Fig. 1. This figure also illustrates that the hot spot is turned towards the observer near phase $\Phi=0.75$ only, as already stated by Peters (2001) for six Algols with a main sequence B-type gainer. Moreover, when the criterion of Lubow \& Shu (1975) shows that the gainer is surrounded by an accretion disk, the point $P$ is located at its edge.

\section{The spin-up of the gainer}

Conservation of angular momentum spins the gainer up due to the impact of RLOFmaterial coming from the donor. Mass located near the gainer's equator gets loosely 


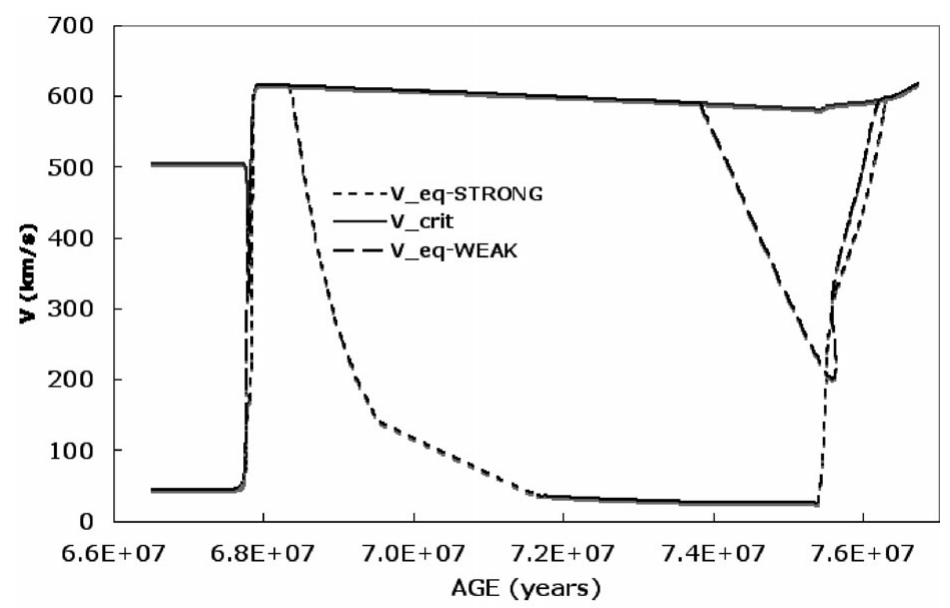

Figure 2. Spin-up of the gainer of a $(6+3.6) M_{\odot}$ binary with an initial period of 3 days.

bound when the gainer rotates rapidly. The spinning-up of the gainer is characterized by an enhancement of its rotational angular momentum $\Delta J_{g}^{+}$which is given in cgs-units by Packet (1981), corrected with the impact-parameter $d$ shown in Fig. 1:

$$
\Delta J_{g}^{+}=6.05 \times 10^{51} \times\left[\frac{R_{g}}{R_{\odot}}\right]^{\frac{1}{2}} \times\left[\frac{M_{g}}{M_{\odot}}+\frac{\Delta M_{g}}{2 M_{\odot}}\right]^{\frac{1}{2}} \times \frac{\Delta M_{g}}{M_{\odot}} \times \frac{d}{R_{g}}
$$

This spin-up is, however, counteracted by tidal interactions which were first studied by Darwin (1879). The formalism for tidal downspinning is taken from Zahn (1977), who gives a suitable approximation for the synchronisation time-scale:

$$
\tau_{\text {sync }}(y r)=q^{-2} \times\left[\frac{a}{R_{g}}\right]^{6}
$$

This expression uses the semi-major axis $a$ of the binary and a mass-ratio $q$, in which the star that has to be synchronized is in the denominator. This is the gainer in our case, so that $q=\frac{M_{d}}{M_{g}}$. Tidal interactions modulate the angular velocity of the gainer $\omega_{g}$ with the angular velocity $\omega_{\text {orb }}$ of the system. According to Tassoul (2000) one can write:

$$
\frac{1}{\omega_{g}-\omega_{\text {orb }}} \times \frac{d \omega_{\text {orb }}}{d t}=-\frac{1}{\tau_{\text {sync }} \times f_{\text {sync }}}=-\frac{1}{t_{\text {sync }}}
$$

Using the moment of inertia $I_{g}$ of the gainer we find the expression which was used by Detmers et al. (2008) in their scenario for liberal evolution of massive close binaries:

$$
\Delta J_{g}^{-}=I_{g} \times\left(\omega_{\text {orb }}-\omega_{g}\right) \times\left[1-e^{\left(\frac{-\Delta t}{\tau_{s y n c} \times f_{s y n c}}\right)}\right]
$$

Tidal interactions spin the gainer down when $\omega_{g}>\omega_{\text {orb }}$. Tides spin the gainer up when $\omega_{g}<\omega_{\text {orb }}$. Weak tidal interactions are represented with $f_{\text {sync }}=1$ whereas $f_{\text {sync }}=$ 0.1 implies strong tides. When the upspinning stops at the end of RLOF, tidal interactions settle the system into a situation with $\omega_{g}=\omega_{\text {orb }}$. Expression (3.4) then implies that $\Delta J_{g}^{-}=\Delta J_{g}^{+}=0$. Synchronisation is achieved and angular momentum remains conserved. One sees in Fig. 2 that the gainer is spun up to critical velocity quickly during the rapid era of mass transfer when core H-burning of the donor is at work. This is the case for slow tidal interaction as well as for a binary undergoing strong tides. During the slow phase of mass transfer the rotation of the gainer synchronizes rapidly in the case 
of strong tidal interaction. In the case of weak tidal interaction, synchronization is never achieved before the onset of RLOF B. The rotation of the gainer spins up again towards critical velocity when a second era of fast mass transfer occurs during H-shell burning of the donor. Rapid rotation favors the gainer to lose mass into interstellar space. The example shown in Fig. 2 turns out to evolve marginally liberal.

\section{The gainer's hot spot}

The temperature of the hot spot is conditioned by that part of the accretion luminosity that can be transformed into radiation. It is customary to define the accretion luminosity by its value that it would have if $L_{1}$ is located at infinity: $L_{a c c}^{\infty}=G \times \frac{M_{g} \times \dot{M}_{d}}{R_{g}}$. The position of $L_{1}$ in a semi-detached binary is however not located at infinity but determined by the geometry shown in Fig. 1. Using the values of the potential energy $U$ in the co-rotating system, the real accretion luminosity for a semi-detached binary is thus given by:

$$
L_{a c c}=U\left(L_{1}\right)-U(P)=D \times L_{a c c}^{\infty} \text { with } 0<D<1
$$

The quantity $D$ is zero for a contact system with a gainer which is not spun up and does not show a hot spot. Values of $D$ are shown in the last column of Table 1 for the 13 semi-detached binaries that were used for the calibration of the hot spot characteristics.

The efficiency of the liberal scenario is further defined by the value of the factor of radiative efficiency $\tilde{K}$ which defines the radiation pressure caused by $L_{a c c}$ in the small surface area of the hot spot. van Rensbergen et al. (2010a) showed that in the case of direct impact on the gainer's equator, the quantity $\tilde{K}$ can be calculated with:

$$
\tilde{K}=\frac{\left[\frac{R_{g}}{R_{\odot}}\right]^{2} \times\left[T_{s p o t}^{4}-T_{e f f, g}^{4}\right]}{\frac{L_{a c c}}{L_{\odot}} \times(5770)^{4}}
$$

There are only 13 systems found in the literature with sufficiently reliable observed data to evaluate the quantity $\tilde{K}$ from relation 4.2 . According to the criterion of Lubow \& Shu (1975) ten systems are direct impact systems, while three others have a transient accretion disk. In the case of the formation of a hot spot on the edge of an accretion disk we have to replace in Eq. (4.2) $R_{g}$ by $R_{d i s k}$. We further replaced $T_{e f f, g}$ by $T_{d i s k}$ for $\beta$ Lyr with an opaque and optically thick accretion disk. Two other systems have a transparent and optically thin accretion disk so that their hot spot is observed as a region with a higher temperature as the underlying stellar surface. Table 1 contains the data for 13 interacting binaries that enable us to find a tentative evaluation of $\tilde{K}$, with a (rather poor) best fit as a function of the total mass of the system. The values mentioned in Table 1 are taken from van Rensbergen et al. (2010b) and references therein.

$$
\tilde{K}=3.9188 \times\left[\frac{M_{d}}{M_{\odot}}+\frac{M_{g}}{M_{\odot}}\right]^{1.645}
$$

\section{The catalog}

The tentative calibration of $\tilde{K}$ shown in Eq. (4.3) luckily differs only slightly from the expression found by van Rensbergen et al. (2010a) with data for 11 interacting binaries. The latter expression was used to construct the catalog. The catalog covers a grid of binaries between 3 and $15 M_{\odot}$ for the initially most massive component, different values 
Table 1. Data used to determine $\tilde{K}$ for 13 semi-detached binaries. The first ten binaries are direct impact systems, the last three lines contain disk systems.

\begin{tabular}{ccccccccc}
\hline System & $M_{d}+M_{g}$ & $R_{d}+R_{g}$ & $T_{\text {eff,d }}$ & $T_{\text {eff,g }}$ & $d M / d t$ & $T_{S p o t}$ & $\tilde{K}$ & $D$ \\
\hline VW Cep & $0.90+0.25$ & $0.93+0.50$ & 5000 & 5200 & $8.769 \mathrm{E}-08$ & 7076 & 6.528 & 0.0445 \\
AR Boo & $0.35+0.90$ & $0.65+1.00$ & 5398 & 5100 & $1.484 \mathrm{E}-07$ & 5539 & 1.064 & 0.0535 \\
CN And & $1.30+0.51$ & $1.43+0.92$ & 6500 & 5911 & $1.215 \mathrm{E}-07$ & 6485 & 8.251 & 0.0261 \\
KZ Pav & $0.80+1.20$ & $1.66+1.50$ & 5000 & 6500 & $2.629 \mathrm{E}-08$ & 7357 & 12.544 & 0.280 \\
V361 Lyr & $1.26+0.87$ & $1.02+0.72$ & 6200 & 4500 & $2.178 \mathrm{E}-07$ & 11021 & 5.185 & 0.156 \\
RT Scl & $1.63+0.72$ & $1.67+1.02$ & 7000 & 4800 & $9.500 \mathrm{E}-08$ & 9300 & 36.363 & 0.0923 \\
CL A ur & $1.35+2.24$ & $2.51+2.58$ & 6323 & 9420 & $1.302 \mathrm{E}-07$ & 10598 & 45.080 & 0.178 \\
U Cep & $1.86+3.57$ & $4.40+2.41$ & 4975 & 11215 & $5.092 \mathrm{E}-07$ & 30000 & 305.631 & 0.577 \\
U Sge & $1.99+5.45$ & $5.64+4.11$ & 5500 & 12250 & $2.036 \mathrm{E}-06$ & 20000 & 49.185 & 0.510 \\
SV Cen & $8.56+6.05$ & $5.90+5.00$ & 14000 & 23000 & $1.626 \mathrm{E}-04$ & 37580 & 211.238 & 0.0297 \\
\hline System & \multirow{2}{*}{$M_{d}+M_{g}$} & \multirow{2}{*}{$R_{d}+R_{\text {disk }}$} & $T_{\text {eff } f, d+T_{\text {eff,g }}} T_{\text {disk }}+T_{\text {edge,disk }}$ & dM/dt & $T_{S p o t}$ & $\tilde{K}$ & $D$ \\
\hline SW Cyg & $0.50+2.50$ & $4.30+3.44$ & $4891+9000$ & $6308+4968$ & $2.130 \mathrm{E}-07$ & 13060 & 106.032 & 0.587 \\
V356 Sgr & $3.00+11.00$ & $13.20+9.07$ & $8600+16500$ & $6174+4299$ & $4.442 \mathrm{E}-07$ & 17050 & 659.326 & 0.561 \\
$\beta$ Lyr & $4.25+14.1$ & $16.70+15.88$ & $13000+28000$ & $18279+8919$ & $3.440 \mathrm{E}-05$ & 22590 & 135.355 & 0.446 \\
\hline
\end{tabular}

for the initial mass ratio and a wide range of initial orbital periods so that cases $\mathrm{A}$ and $\mathrm{B}$ are well represented. The evolution of every binary was calculated so as to allow mass to leave the system when the added energy of rapid rotation and radiation from a hot spot exceeds the binding energy of matter located in the hot spot. This situation can only occur during epochs of rapid RLOF, when the mass transfer rate exceeds a well defined critical value. The quantity $\beta$, defined in Eq. (1.1), is thus time dependent. It equals unity most of the time, but can become small during eras of fast mass transfer.

The updated catalog contains 561 conservative and liberal evolutionary tracks and is available at the Centre de Données Stellaires (CDS). Binaries with an initial primary mass $\in[3-5] M_{\odot}$ are calculated in one mode only since they evolve conservatively. Binaries with an initial primary mass $\in[6-15] M_{\odot}$ are calculated in the liberal mode. Results for evolution with weak and strong tidal interaction are given separately. Conservative tracks are always added so that the reader is able to compare results of liberal (in two different tidal modes) and conservative evolution.

\section{References}

Darwin, G. 1879, Philosophical Transactions of the Royal Society of London, 170, 447

De Loore, C. \& De Greve, J. P. 1992, A\&̈AS, 94, 453

Detmers, R. G., Langer, N., Podsiadlowski, P., \& Izzard, R. G. 2008, A\& $A, 484,831$

Kippenhahn, R. \& Weigert, A. 1967, ZfA, 65, 251

Kippenhahn, R., Kohl, K., \& Weigert, A. 1967, ZfA, 66, 58

Lubow, S. H. \& Shu, F. H. 1975, ApJ, 198, 383

Mennekens, N., Vanbeveren, D., De Greve, J. P., \& De Donder, E. 2010, A\&̊A, 515, A89

Meurs, E. J. A. \& van den Heuvel, E. P. J. 1989, A\&A, 226, 88

Nelson, C. A. \& Eggleton, P. P. 2001, ApJ, 552, 664

Packet, W. 1981, A\&A, 102, 17

Paczyński, B. 1967a, AcA, 17, 193

Paczyński, B. 1967b, AcA, 17, 355

Peters, G. J. 2001, in: D. Vanbeveren (eds.), The Influence of Binaries on Stellar Population Studies, Astrophysics and Space Science Library 264, p. 79

Podsiadlowski, P., Joss, P. C., \& Hsu, J. J. L. 1992, ApJ, 391, 246

Tassoul, J.-L. 2000, Stellar Rotation, Cambridge University Press (New York)

van Rensbergen, W., De Greve, J. P., De Loore, C., \& Mennekens, N. 2008, A\&\&A, 487, 1129 
van Rensbergen, W., De Greve, J. P., Mennekens, N., Jansen, K., \& De Loore, C. 2010a, A\&A, $510, \mathrm{~A} 13$

van Rensbergen, W., De Greve, J.-P., Mennekens, N., Jansen, K., \& De Loore, C. 2010b, A\&̈A 528, A 16

Wellstein, S., Langer, N., \& Braun, H. 2001, A\&A, 369, 939

Zahn, J.-P. 1977, A\&SA, 57, 383

\section{Discussion}

Koeningsberger: Are both the mass-donor and mass-gainer rotating synchronously? If not, how did you deal with rotation?

VAN Rensbergen: The binary is born with well defined values of masses, radii and orbital periods. Before RLOF starts, there is time to synchronize the system. But after the onset of RLOF, the rotation of the gainer is modulated by spin-up through mass impacting from the donor and spin-down due to tidal interaction. We did not (although we should) take the enhanced rotational velocity of the gainer into account to calculate modifications of its internal structure. Neither did we follow up the rotation of the donor.

Myron Smith: I would like to raise your attention to the small group of $\gamma$ Cas stars. This is an important group of Be stars known for their hard X-ray emissions. $\gamma$ Cas itself is a widely spaced ( $\mathrm{P}=504$ days, $\mathrm{e} \approx 0)$ binary, and two others are arguably blue stragglers. It would be of great interest to know what the products are in terms of the secondary stars. Are these degenerate products white dwarfs, neutron stars or something else? A grid of evolutionary models of binary systems would provide important checks as to how these strange systems have come to be.

VAn REnsBergen: Binaries with short initial orbital periods are followed up until overcontact when both stars are still on the main sequence. The evolution of systems with longer initial periods is only calculated until exhaustion of He in the core of the donor. From that moment on further evolution can be predicted. Implementing the data of our catalog into the population code used by Mennekens et al. (2010), we find that binaries with an initial mass of the primary below $7 M_{\odot}$ eventually yield many WD+WD systems. Binaries with one or two neutron stars can be expected from the evolution of the most massive systems in our catalog.

MATHIs: You speak about two tidal interactions (the weak and the strong tidal interactions). Could you give more details about this?

Van Rensbergen: For the spin-up of the gainer we used the formalism of Packet (1981), including the fact that the effect diminishes strongly when the orbit of the semi-detached binary is narrow. The spin-down is settled by tidal interaction as formulated in principle by Darwin (1879), treated rigorously in his book on stellar rotation by Tassoul (2000) and modulated by Zahn (1977) who makes a difference between strong and weak tidal interaction. We calculated the evolution of binaries using both types of tides. Although we found some significant individual differences when the mass loss from the system is large, we also found that the global result (e.g. distribution of mass ratios and orbital periods of Algols) remains very much the same. 